\title{
Implementation of an Evidence-Based Exercise Program for Older Adults in South Florida
}

\author{
Richard C. Palmer, ${ }^{1}$ Anamica Batra, ${ }^{1}$ Chelsie Anderson, ${ }^{1}$ Timothy Page, ${ }^{1}$ \\ Edgar Vieira, ${ }^{2}$ and Laura Seff ${ }^{1}$ \\ ${ }^{1}$ Robert Stempel College of Public Health and Social Work, 11200 SW 8th Street, Miami, FL 33199, USA \\ ${ }^{2}$ College of Nursing and Health Sciences, 11200 SW 8th Street, Miami, FL 33199, USA
}

Correspondence should be addressed to Richard C. Palmer; richard.palmer@fiu.edu

Received 23 May 2016; Revised 31 August 2016; Accepted 14 September 2016

Academic Editor: Elke Bromberg

\begin{abstract}
Copyright (C) 2016 Richard C. Palmer et al. This is an open access article distributed under the Creative Commons Attribution License, which permits unrestricted use, distribution, and reproduction in any medium, provided the original work is properly cited.
\end{abstract}

\begin{abstract}
Introduction. This study aimed to examine how well an evidence-based physical activity program could be translated for wide scale dissemination and adoption to increase physical activity among community-dwelling older adults. Methods. Between October 2009 and December 2012, reach, fidelity, dosage, ease of implementation, and barriers to translation of EnhanceFitness (EF) were assessed. To assess effectiveness, a pretest-posttest design was used to measure increases in functional fitness (chair stands, arm curls, and the up-and-go test). Results. Fourteen community-based agencies offered 126 EF classes in 83 different locations and reached 4,490 older adults. Most participants were female (72\%). Thirty-eight percent of participants did not complete the initial 16-week EF program. The $25 \%$ who received the recommended dose experienced an increase in upper and lower body strength and mobility. Further, participants reported high satisfaction with the program. Conclusion. EF was successfully implemented in a variety of settings throughout South Florida and reached a large number of older adults. However, challenges were encountered in ensuring that those who participated received a program dose that would lead to beneficial gains in functional fitness.
\end{abstract}

\section{Introduction}

Regular physical activity can promote physical and psychological wellbeing and reduce risk of disability and vulnerability to chronic diseases in older adults [1-5]. Nevertheless, adults over 50 years of age are the least physically active segment of the US adult population [6-8]. Evidence-based exercise programs designed for older adults show promise as a way to increase opportunities for safe and effective physical activity [9]. However, little is known about how well these programs, which were tested and evaluated using controlled trials, are translated for wider use in community settings [10]. It is important to know if research-based programs will maintain their effectiveness when they are implemented in community settings [11]. Further, accurate interpretation of outcomes depends on knowing which aspects of the intervention were delivered and how faithful the delivery was to original program design [12].
The Healthy Aging Regional Collaborative (HARC) was established by the Health Foundation of South Florida (HFSF) to increase the adoption of evidence-based programs that promote and preserve the health of older adults in South Florida. Among the programs selected was EnhanceFitness $(\mathrm{EF})$, which is an evidence-based group exercise program for older adults of all fitness levels [13, 14]. EF aims to help participants improve overall functional fitness and personal wellbeing. Intervention studies have documented the efficacy of EF and have shown that EF improves performance on fitness assessments, perceived physical functioning, social functioning, mental health, and decreases bodily pain [1518]. Limited evidence exists demonstrating that EF can be translated to community-based settings and still achieve improvements in fitness and wellbeing [19]. Therefore, we investigated the ability of HARC to implement and sustain the EF program in South Florida over a three-year period. We examine if HARC was able to retain program fidelity, 
recommended dosage and exposure to the program, and comparable gains in functional fitness.

\section{Methods}

2.1. Setting. The Healthy Aging Regional Collaborative was created to increase local community capacity to offer accessible health promotion programs to older adults in South Florida. HARC was established by the HFSF and was designed to (1) disseminate evidence-based health promotion programs to address inactivity and chronic health conditions in older adults in South Florida; (2) establish a local group of trained professionals and lay leaders with the capacity to provide a range of evidence-based health promotion programs to older adults on an ongoing basis; and (3) provide a foundation for planning, coordination, technical assistance, and peer learning for present and future collaborative members. Community-based agencies that were interested in participating in HARC had to apply to HFSF and received funding to offer evidence-based programs, including EF. Funding covered licensing for use of the selected evidencebased program, instructor training, program implementation materials and equipment, and support for the administrative costs of implementation, including procurement of sites, instructors and participants, data collection, and data entry. Funded agencies were responsible for delivering EF. Agencies identified suitable sites to offer EF classes (i.e., churches, senior housing, senior centers, community centers, and parks) as well as recruited program participants.

2.2. EnhanceFitness Overview and Implementation. A onehour EF class includes a 5-minute warm-up, a 20-minute low impact aerobic workout, a 5-minute cooldown, a 20-minute strength training workout, and 10 minutes of stretching and is offered three times per week. Instructors who were certified by a nationally recognized fitness organization (i.e., YMCA, $\mathrm{ACE}$, or ACSM) and who received training in EF procedures at a two-day training conducted by EF program developers and EF master trainers led classes. All instructors were required to teach $\mathrm{EF}$ as outlined in $\mathrm{EF}$ training materials provided by the program developer. Individuals over the age of 50 who were interested in participating in EF were enrolled by agencies as long as they were cognitively and physically able to participate in the EF program.

2.3. Data Collection and Management. Multiple sources of data were collected to evaluate how well HARC agencies were able to implement EF. Descriptive/demographic data form, a health history, a fitness check (FC), and program satisfaction survey were collected. Participant data were collected at enrollment, with a second FC and the evaluation scheduled to be completed 16 weeks after enrollment as prescribed by the EF program. Program managers and instructors collected all participant data. All staff who administered the forms and collected data were trained regarding the meaning and importance of the items and shown the correct way to administer the three functional tests included on the FC during EF training sessions. Program managers submitted class registrations, new and ongoing participant data, and participant attendance records to HARC's evaluation team. An annual survey of program managers was also conducted online. Program fidelity was monitored by trained observers who observed randomly selected classes and then completed a fidelity observation checklist aimed at identifying implementation issues.

Prior to receiving any data from agencies, HARC's evaluation team received approval from the Institutional Review Board at Florida International University to conduct the evaluation of HARC.

2.4. Measures. The number of agencies, sites, classes, and participants were tracked as measures of EF implementation. Demographic characteristics of participants were selfidentified on a form that included questions regarding gender, age, race and ethnicity, highest level of education, annual personal income, living situation (alone or with others), marital status, and primary spoken language. Participant satisfaction was measured using the satisfaction survey developed and provided by EF.

Effectiveness of the EF program was assessed based on changes in measures of functional fitness (30-second arm curl to assess upper extremity strength, the 30-second chair stand to measure lower extremity strength, and the timed eightfoot up-and-go circuit to measure balance and mobility), selfreported general health, and self-reported frequency (days per week) of exercise of at least 30-minute duration.

Additional measures included (1) the BRFSS general health measure, with a response scale from $1=$ poor to $5=$ excellent [20]; (2) self-reported number of days of exercise of at least 30-minute duration in a week, including EF sessions; and (3) a single item on the second FC to measure the self-reported degree of improvement in physical abilities (walking, bending, strength, moving around, and doing the activities you want to do) as a result of EF program participation, reported on a 10-point scale where 1 is no improvement and 10 is great improvement. Attendance records provided data regarding dose, average number of sessions attended by participants, and average duration of participant enrollment.

The annual survey of HARC program coordinators served as a process evaluation tool and identified implementation issues that agencies in HARC experienced. Questions specific to implementing EF were included each year and only agencies who offered the EF program completed these items. Items assessed ease of implementing the EF program, challenges with implementation, assessed participant recruitment activities, and also assessed long-term adoption and sustainability.

Observations of $30 \%$ of randomly selected classes assessed fidelity to the program design. Observers were staff members of the evaluation team and were trained on program specifications through a detailed review of the instructor manual. As part of the training, observers would accompany an experienced observer and interrater reliability would be assessed. Observers used a structured checklist that was based on the instructor manual and was developed by the evaluation team, EF master trainers, and HARC member agencies. 


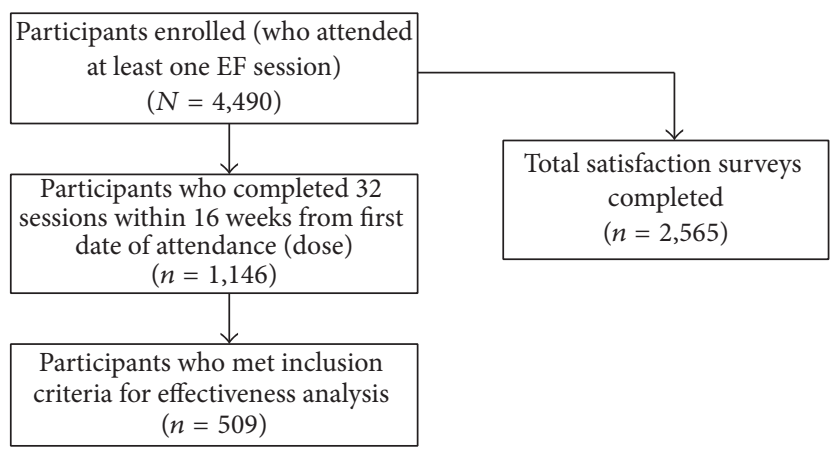

FIGURE 1: Number of participants by inclusion criteria.

The checklist specified observable elements related to the class environment, instructor, duration, and site. Each element was marked as observed or not observed. Discrepancies from the prescribed exercise routine were noted in a comment section.

2.5. Data Analysis. Of the 4,490 enrolled participants, 1,146 received the dose of the intervention proposed by Belza and colleagues [13] (Figure 1). Exclusion criteria for the current study include (1) no data for the pretest, the posttest, or both; (2) a first FC that did not fall within 30 days before and seven days after the first attendance date (to assure that baseline data reflected actual abilities prior to any benefits received from attending EF sessions); (3) a second FC completed before the recommended dose was attained; and (4) more than six months between the first and second fitness checks. With these exclusions, the sample used to calculate participant outcomes included 509 participants (Figure 1).

Data were extracted from the online database and imported into SPSS 22.0 for analysis. Data was first assessed for normality and outliers. Data on participant satisfaction, self-reported improvement in physical abilities, participant characteristics, participant attendance metrics, and program manager survey and fidelity observation data were analyzed using descriptive statistics. To test specific hypotheses about program effectiveness, the general linear model (GLM) was chosen to assess within-subject changes in outcome measures (arm curls, chair stands, and 8-foot up-and-go) at baseline and 6 weeks and controlled for potential differences in performance site [21].

\section{Results}

A total of 4,490 older adults enrolled and attended at least one EF session between October 1, 2009, and December 31, 2012. The majority of enrolled participants were women. Participant ages ranged from 29 to 107, with a mean age of 74.8 years $(\mathrm{SD} \pm 9.9)$. The racial/ethnic composition of participants was diverse, with non-Hispanic whites and Hispanics making up the two largest groups (Table 1). The number of agencies, classes, sites, and participants, tracked in each of the first three years of the HARC as measures of EF implementation, are shown in Table 2, by year. Total number of participants reached increased every year. The largest number of new participants was reached in Year 1. After an expected reduction of new participants in Year 2, the number of new participants increased in Year 3, but not to the Year 1 level. At the end of the three-year period, nine agencies were funded and active, offering 70 ongoing EF classes at 56 sites (Table 2).

3.1. Fidelity. Sixty-nine EF classes were observed for fidelity by the evaluation team between, accounting for nearly $33 \%$ of all EF classes offered during the time period. Almost half of the observed classes, $47.8 \%$, had no significant fidelity concerns in the measured dimensions. The most frequently cited concerns related to instructors not using key phrases developed to ensure participant safety, including checking if participants could see and hear the instructor (30\%), and reminding participants not to do exercises their physician advised against (30\%), not to do any exercises that were painful (16\%), and to exercise at a pace that was comfortable (14\%). Additionally, $41 \%$ of observed sessions lasted less than 60 minutes (Figure 2).

3.2. Dose. Of 4,490 participants enrolled in the HARC EF program between October 1, 2009, and December 31, 2012, who attended at least one session, an average of $26 \%$ received the minimum recommended dose [13] (Table 2). The ratio of completers to new participants enrolled declined in each of the three years. Other measures, potentially relevant in terms of dose, were examined. Three percent of enrolled participants attended only one session and 38\% stopped attending within the initial 16 weeks. The average time between enrollment and last date of attendance was 1.1 years. The average number of sessions attended per participant was 59.

3.3. Ease of Implementation and Barriers. In the Year 1 program manager survey, six of seven program managers responsible for implementing EF reported that implementation was difficult or very difficult on a five-point scale from very easy to very difficult. In the Year 2 and Year 3 surveys, more than half of the program managers indicated that implementation was moderate, that is, half-way between very easy and very difficult on the measurement scale, showing 
TABLE 1: Characteristics of EnhanceFitness participants.

\begin{tabular}{|c|c|}
\hline & $N=4,490^{\mathrm{a}}$ \\
\hline \multicolumn{2}{|l|}{ Gender } \\
\hline Female & 3,216 \\
\hline Male & 862 \\
\hline \multicolumn{2}{|l|}{ Age } \\
\hline$<60$ years & 176 \\
\hline $60-69$ years & 948 \\
\hline $70-79$ years & 1,462 \\
\hline $80-89$ years & 936 \\
\hline 90 years and over & 276 \\
\hline \multicolumn{2}{|l|}{ County of residence } \\
\hline Broward & 1,588 \\
\hline Miami-Dade & 1,920 \\
\hline Monroe & 645 \\
\hline \multicolumn{2}{|l|}{ Race-ethnicity } \\
\hline Hispanic/Latino & 677 \\
\hline Haitian/other non-Hispanic Caribbeans & 144 \\
\hline White, non-Hispanic & 794 \\
\hline Black, non-Haitian & 188 \\
\hline Other & 44 \\
\hline \multicolumn{2}{|l|}{ Marital status } \\
\hline Married/partnered & 1,625 \\
\hline Single/not partnered & 2,343 \\
\hline \multicolumn{2}{|l|}{ Annual income } \\
\hline$<\$ 15,000$ & 1,089 \\
\hline$\$ 15,000-\$ 24,999$ & 450 \\
\hline$\$ 25,000-\$ 49,999$ & 404 \\
\hline$\$ 50,000-\$ 75,000$ & 185 \\
\hline$>\$ 75,000$ & 151 \\
\hline \multicolumn{2}{|l|}{ Education } \\
\hline Less than high school & 613 \\
\hline High school graduate & 919 \\
\hline Some college & 1,031 \\
\hline College graduate or higher & 1,404 \\
\hline \multicolumn{2}{|l|}{ Other variables } \\
\hline Frail/disabled & 470 \\
\hline Has Medicare & 2,641 \\
\hline Has Medicaid & 807 \\
\hline
\end{tabular}

$\overline{{ }^{a} \text { Reported values may not add up to total participant } N \text { due to missing data. }}$

that ease of implementation improved over time. Implementation barriers most frequently cited over the three years remained relatively constant and included funding, inadequate staff, inadequate client interest, cultural norms of the community, and difficulty in recruiting class instructors. The most frequent barriers identified for recruiting participants included difficulty maintaining consistent participation and the seasonal residence of some participants in the South Florida area. The most frequent barriers to instructor recruitment included availability for the three weekly one-hour sessions, finding individuals who were committed to the EF program, and lack of transportation.
3.4. Effectiveness. Analysis revealed that participants who completed the recommended dose and received a posttest after participating in the program for four months significantly improved their scores on the three physical ability measures, self-reported general health, and frequency of daily exercise of at least 30-minute duration after participating in EF program (Table 3).

3.5. Participant Satisfaction. When asked on the second FC about improvements in physical abilities as a result of their EF participation, $71.5 \%$ of participants indicated improvement. Among the 2,565 EF participants who completed a program evaluation, $89 \%$ were satisfied or highly satisfied with the program. Additionally, 97\% responded that they would take the class again and $92 \%$ would recommend the class to a friend.

\section{Discussion}

The HARC initiative described here is the largest scale translation of EF to a broad cross section of community agencies and settings within the same geographical area to date in the United States. Data reported here suggest that HARC was able to successfully implement EF throughout South Florida and reach a large number of older adults over the threeyear period. These findings indicate that this effort to form a collaborative, sharing resources, training, and knowledge, was an effective approach for translating an evidence-based program to a wide array of community settings and sites.

Unlike controlled trials of EF, data reported here are based on the real world implementation of EF in a variety of community settings throughout South Florida. It was important to investigate if the community-based EF implementation produced participant changes similar to those reported in controlled EF efficacy studies. Our findings are consistent with prior controlled studies of EF and other fitness programs $[13,15,16]$. Participants in this study demonstrated statistically significant improvements in upper body strength, lower body strength, functional mobility, self-reported health status, and frequency of daily exercise of at least 30-minute duration over the follow-up period.

As with any adoption of a health promotion program, the potential for changes and deviations in the curriculum is a concern. Adaptation is a normal process, yet it can cause a program to be less effective if changes are made that undermine the program's effectiveness. In our evaluation, we assessed fidelity to determine how accurately EF agencies and instructors implemented the program. Surprisingly, very few concerns were identified for the core elements of the program. The majority of observed concerns involved safety warnings that instructors are supposed to use throughout the class.

Our evaluation did identify several areas that warrant comment and further investigation. A participant recruitment challenge relating to male participants existed. Only about $15 \%$ of participants were male, while the percentage of males in the total population in the three targeted Florida counties is much higher, at nearly $44 \%$ [22]. This rate of male participation is similar to findings of other programs 
TABLE 2: HARC EnhanceFitness process measures.

\begin{tabular}{|c|c|c|c|c|}
\hline Measure & $\begin{array}{c}\text { Year } 1 \\
N\end{array}$ & $\begin{array}{c}\text { Year } 2 \\
N\end{array}$ & $\begin{array}{c}\text { Year } 3 \\
N\end{array}$ & $\begin{array}{c}\text { All years } \\
N\end{array}$ \\
\hline Participants & 1,528 & 1,961 & 2,127 & 4,490 \\
\hline Percent completers & $29.4 \%$ & $24.3 \%$ & $21.8 \%$ & $25.5 \%$ \\
\hline Agencies offering EF classes & 8 & 9 & 9 & 14 \\
\hline EF classes offered & 59 & 73 & 79 & 126 \\
\hline Classes observed for fidelity & 18 & 25 & 26 & 69 \\
\hline Sites where EF classes were offered & 51 & 60 & 63 & 83 \\
\hline
\end{tabular}

EF: EnhanceFitness.

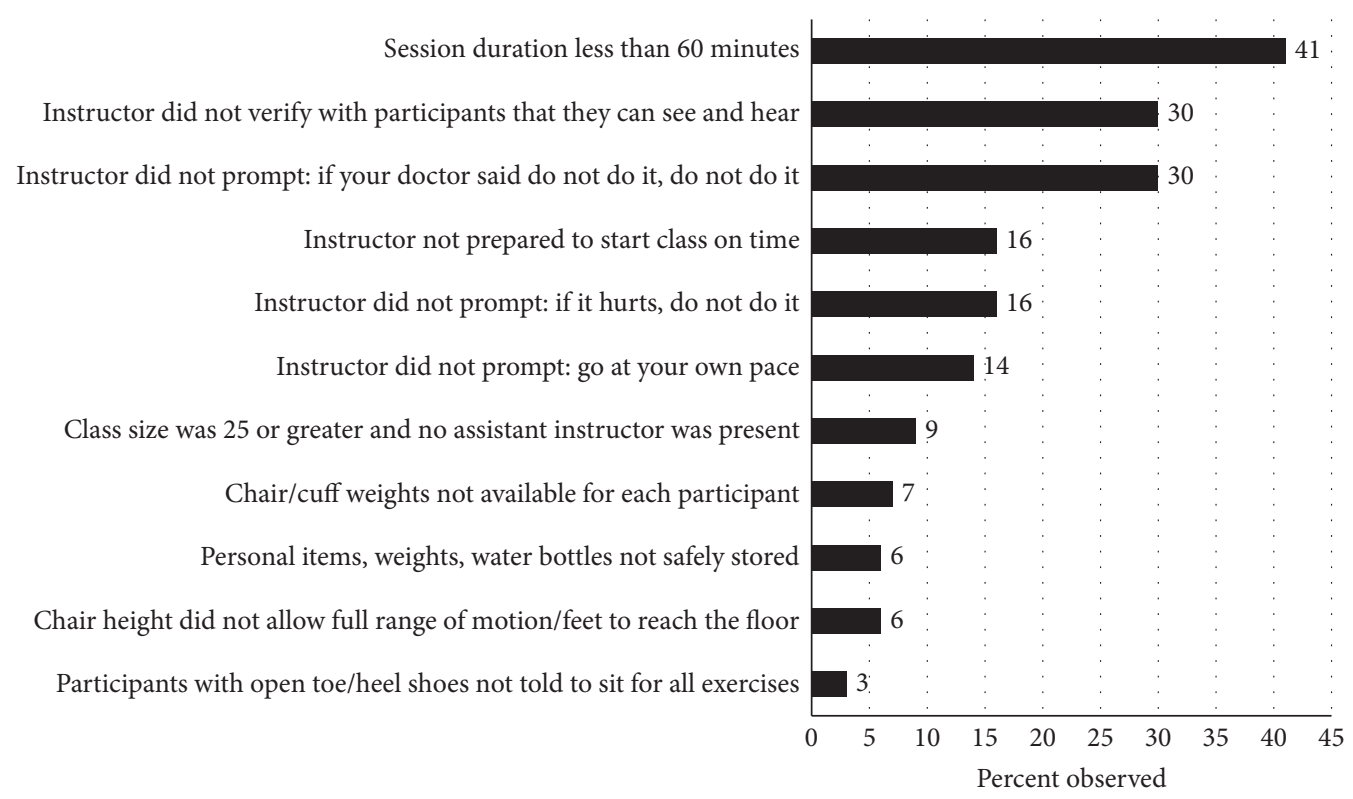

FIGURE 2: Frequency of fidelity concerns identified.

that serve older adults, although there is limited knowledge regarding why older men do not participate in health promotion programs $[23,24]$. There is a need for research that explores how to overcome male resistance to such participation. Additionally, of the process issues identified, perhaps the most significant were frequency of attendance and attrition. There appears to be a problem with participants achieving the recommended dosage (32) sessions within a 16week period that has been associated with functional fitness and mobility benefits [13]. Almost $41 \%$ of all EF participants in this region stopped attending within the initial 16-week period. Moreover, for those who did continue through the initial 16 weeks and beyond, only $26 \%$ attended at least 32 sessions in the first 16 weeks. In both cases, participants did not receive the dose that has been suggested as being optimal to obtain all the benefits associated with exercise and strength training. For EF to be effective, participants must participate regularly to achieve maximum benefits. Subsequently, more research is warranted to better understand the elements that affect attendance patterns of older adults at ongoing exercise programs and to explore strategies that effectively address these attendance barriers.
Although findings show that EF can be effectively implemented by a collaborative in South Florida and that participants can achieve benefits consistent with clinical trials if they participate regularly, this evaluation has certain limitations. As this was a community-based implementation and not a controlled research study, a randomized design could not be used. Participants self-selected into the program, so they may not be representative of the larger older adult population in South Florida. Bias could also be an issue if functional test results reflect the impact of health-promoting activities that participants engaged in other than EF. A large number of participants did not have documented follow-up measures, which reduced data available for analysis of fitness test results. Finally, functional test data are potentially subject to measurement bias because different instructors collected data on outcome measures. However, the rigorous training on data collection tools prior to being certified to teach an EF class, use of the same tool, and ongoing fidelity monitoring of instructors reduces the chances of measurement bias.

Even with these limitations, this study had strengths. The program was implemented by multiple agencies, and the diverse racial/ethnic mix of the South Florida participants is 
TABLE 3: EnhanceFitness effectiveness measures.

\begin{tabular}{llccr}
\hline Fitness test & $N^{\mathrm{a}}$ & $\begin{array}{c}\text { Baseline } \\
\text { mean (SD) }\end{array}$ & $\begin{array}{c}\text { Follow-up } \\
\text { mean (SD) }\end{array}$ & Change \\
\hline Number of chair stands in 30 seconds & 491 & $11.78(4.09)$ & $15.41(5.36)$ & 3.63 \\
Number of arm curls in 30 seconds & 499 & $15.92(5.85)$ & $20.76(7.34)$ & 4.84 \\
Number of seconds to complete up-and-go & 487 & $8.62(4.69)$ & $7.40(4.28)$ & -1.22 \\
\hline
\end{tabular}

SD: standard deviation; N/A: not applicable.

${ }^{\mathrm{a}}$ Reported values do not add up to total participant $(N=509)$ due to missing data.

likely different than demographic profiles found in previously described evaluations. Many measures of implementation used indicated successful introduction of the programs and ongoing fidelity to program design. A longer-term retrospective study of sustained fidelity and the program's impact on participant physical fitness, health status, frequency of exercise, and healthcare costs is needed to confirm that outcomes reported here endure over longer time periods.

\section{Conclusions}

Health promotion efforts targeting older adults are essential for improvement in quality of life, functional ability, and healthcare cost savings. HARC's innovative approach to rapid EF implementation on a wide scale shows that EF can be introduced in many different settings and delivered successfully. Moreover, there is reason to believe that communitybased EF programs have the potential to offer access to an evidence-based physical activity with proven health benefits to a large percentage of older adults in the community. However, efforts are needed to ensure that community-based agencies that offer EF reinforce the importance of continued attendance to ensure the most benefit to program participants and that implementers continue to assess implementation fidelity of the program to the original design tested in controlled trials.

\section{Competing Interests}

The authors declare that there are no competing interests regarding the publication of this manuscript.

\section{Acknowledgments}

This study was supported by the Health Foundation of South Florida as part of the Healthy Aging Initiative. The authors would like to thank the member agencies of the Healthy Aging Regional Collaborative for their support and assistance with program implementation and data collection.

\section{References}

[1] US Department of Health and Human Services, 2008 Physical Activity Guidelines for Americans Summary, 2008, https:// health.gov/paguidelines/guidelines/summary.aspx.

[2] M. E. Cress, D. M. Buchner, T. Prohaska et al., "Physical activity programs and behavior counseling in older adult populations,"
Medicine \& Science in Sports \& Exercise, vol. 36, no. 11, pp. 19972003, 2004.

[3] R. S. Mazzero, P. Cavanagh, J. Evans et al., "American College of Sports Medicine. Position stand: exercise and physical activity for older adults," Medicine \& Science in Sports \& Exercise, vol. 30, no. 6, pp. 992-1008, 1998.

[4] W. J. Rejeski and S. L. Mihalko, "Physical activity and quality of life in older adults," Journals of Gerontology Series A: Biological Sciences and Medical Sciences, vol. 56, no. 2, pp. 23-35, 2001.

[5] F. Sun, I. J. Norman, and A. E. While, "Physical activity in older people: a systematic review," BMC Public Health, vol. 13, article 449, 2013.

[6] J. Kruger, S. A. Carlson, and D. Bucher, "How active are older Americans?" Preventing Chronic Disease, vol. 4, pp. 1-12, 2007.

[7] Centers for Disease Control and Prevention, Behavioral Risk Factor Surveillance System Survey Data, U.S. Department of Health and Human Services, Centers for Disease Control and Prevention, Atlanta, Ga, USA, 2006.

[8] C. A. Schoenborn and K. M. Heyman, "Health characteristics of adults aged 55 years and over: United States, 2004-2007," National Health Statistics Reports 16, National Center for Health Statistics, Hyattsville, Md, USA, 2009, http://www.cdc .gov/nchs/data/nhsr/nhsr016.pdf.

[9] A. C. King, W. J. Rejeski, and D. M. Buchner, "Physical activity interventions targeting older adults. A critical review and recommendations," American Journal of Preventive Medicine, vol. 15, no. 4, pp. 316-333, 1998.

[10] R. E. Glasgow, E. Lichtenstein, and A. C. Marcus, "Why don't we see more translation of health promotion research to practice? Rethinking the efficacy-to-effectiveness transition," American Journal of Public Health, vol. 93, no. 8, pp. 1261-1267, 2003.

[11] L. Dusenbury, R. Brannigan, W. B. Hansen, J. Walsh, and M. Falco, "Quality of implementation: developing measures crucial to understanding the diffusion of preventive interventions," Health Education Research, vol. 20, no. 3, pp. 308-313, 2005.

[12] J. A. Durlak and E. P. DuPre, "Implementation matters: a review of research on the influence of implementation on program outcomes and the factors affecting implementation," American Journal of Community Psychology, vol. 41, no. 3-4, pp. 327-350, 2008.

[13] B. Belza, S. Snyder, M. Thompson, and J. LoGerfo, "From research to practice: EnhanceFitness, an innovative community-based senior exercise program," Topics in Geriatric Rehabilitation, vol. 26, no. 4, pp. 299-309, 2010.

[14] Senior Services, Enhance Fitness, 2013, http://www.projectenhance.org/EnhanceFitness.aspx.

[15] T. L. Moore-Harrison, M. A. Johnson, M. E. Quinn, and M. E. Cress, "An evidence-based exercise program implemented in congregate-meal sites," Journal of Physical Activity and Health, vol. 6, no. 2, pp. 247-251, 2009. 
[16] S. Snyder and B. Belza, "Eliminating disparities in communities of color through the Lifetime Fitness Program," 2005, http://www.cdc.gov/pcd/issues/2005/apr/04_0142j.htm.

[17] B. Belza, A. Shumway-Cook, E. A. Phelan, B. Williams, S. J. Snyder, and J. P. LoGerfo, "The effects of a community-based exercise program on function and health in older adults: the EnhanceFitness program," Journal of Applied Gerontology, vol. 25, no. 4, pp. 291-306, 2006.

[18] J. I. Wallace, D. M. Buchner, L. Grouthaus et al., "Implementation and effectiveness of a community-based health promotion program for older adults," Journals of Gerontology Series A: Biological Sciences and Medical Sciences, vol. 53, pp. M301M306, 1998.

[19] M. Tomioka, N. Sugihara, and K. L. Braun, "Replicating the enhancefitness physical activity program in Hawai'i's multicultural population, 2007-2010," Preventing Chronic Disease, vol. 9, no. 3, Article ID 110155, 2012.

[20] Centers for Disease Control and Prevention, Behavioral Risk Factor Surveillance System Survey Questionnaire, U.S. Department of Health and Human Services, Centers for Disease Control and Prevention, Atlanta, Ga, USA, 2008.

[21] P. McCullagh and J. Nelder, Generalized Linear Models, Chapman and Hall/CRC, London, UK, 2nd edition, 1989.

[22] U.S Census Bureau, 2010 Census Data, http://www.census.gov/ 2010census/data/.

[23] W. H. Courtenay, "Constructions of masculinity and their influence on men's well-being: a theory of gender and health," Social Science and Medicine, vol. 50, no. 10, pp. 1385-1401, 2000.

[24] M. E. Addis and J. R. Mahalik, "Men, masculinity, and the contexts of help seeking," American Psychologist, vol. 58, no. 1, pp. 5-14, 2003. 


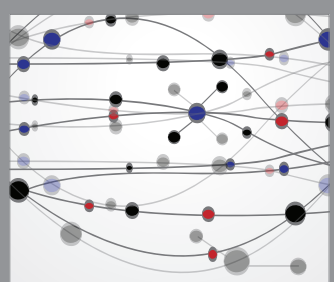

The Scientific World Journal
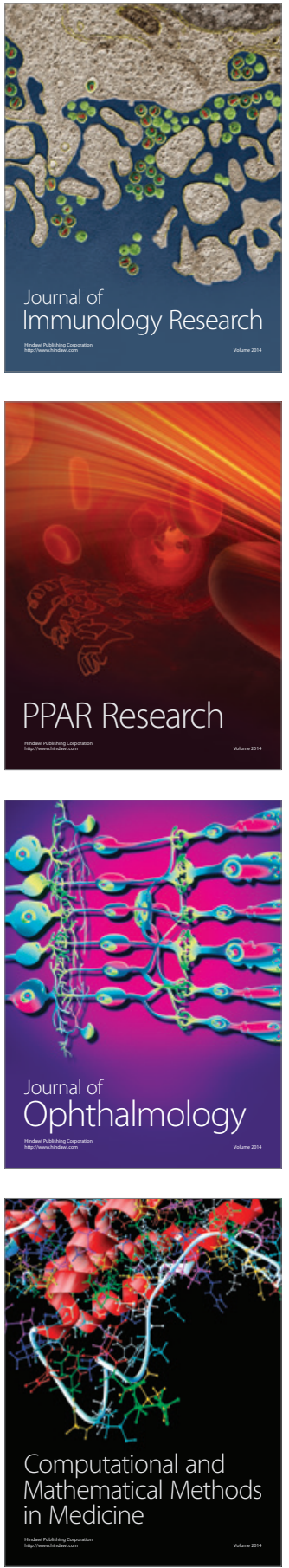

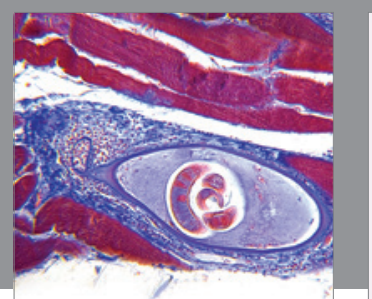

Gastroenterology Research and Practice

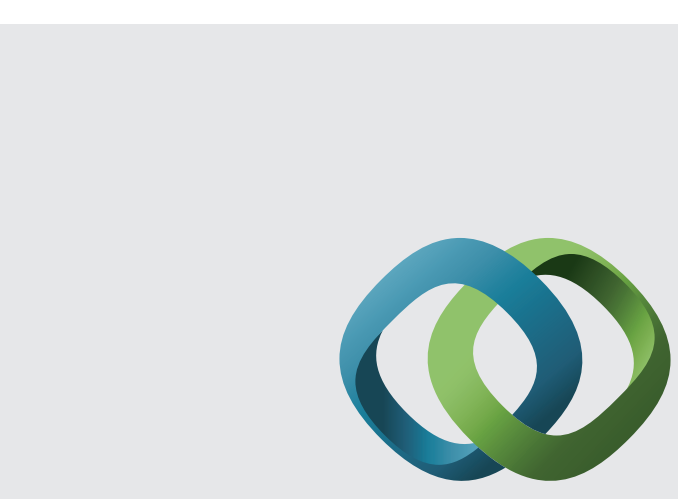

\section{Hindawi}

Submit your manuscripts at

http://www.hindawi.com
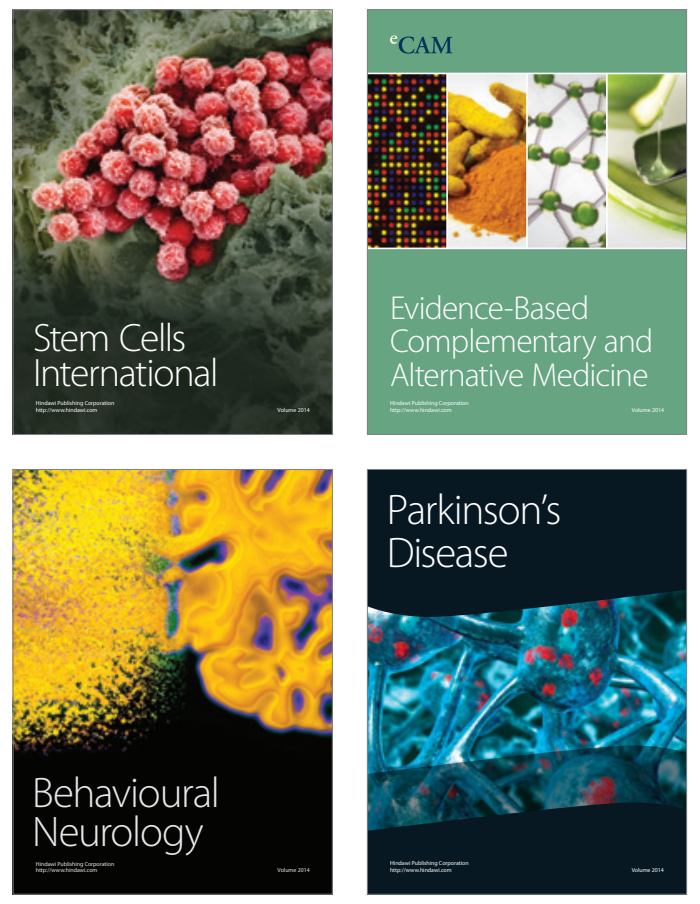
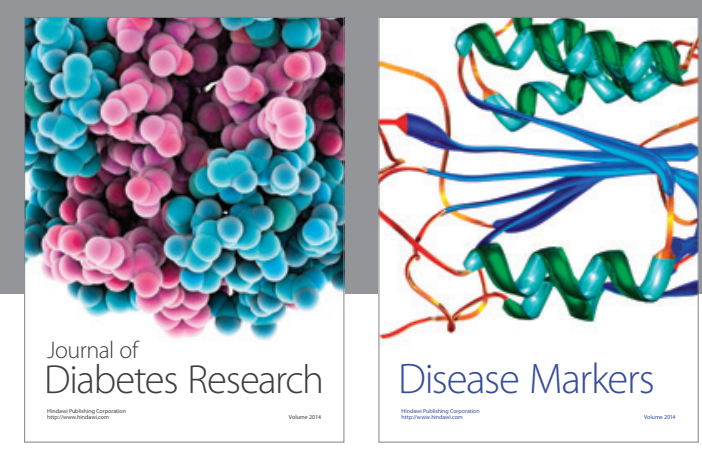

Disease Markers
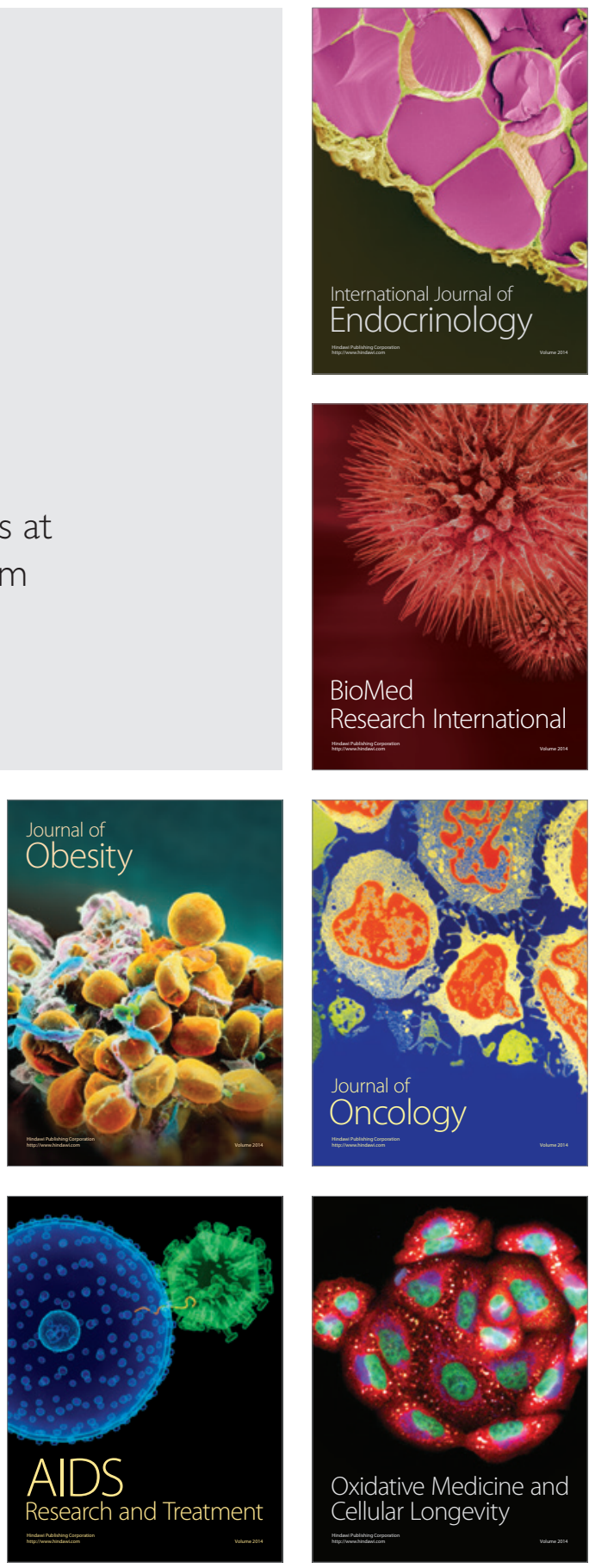